Original Article

\title{
Effects of posture on chest-wall configuration and motion during tidal breathing in normal men
}

\author{
Sachie Takashima, RPT, MS ${ }^{1 *}$, Masafumi Nozoe, RPT, PhD ${ }^{1)}$, Kyoshi Mase, RPT, PhD ${ }^{1)}$, \\ Yusuke Kouyama, RPT ${ }^{2)}$, Kazuhiro Matsushita, RPT ${ }^{3)}$, Hiroshi Ando, MD, PhD $^{4}$ \\ 1) Department of Physical Therapy, Faculty of Nursing and Rehabilitation, Konan Women's University: \\ Kobe, Hyogo, Japan \\ 2) Department of Rehabilitation, Takarazuka City Hospital, Japan \\ 3) Department of Rehabilitation, Hyogo College of Medicine Sasayama Medical Center, Japan \\ 4) Department of Rehabilitation Science, Kobe University Graduate School of Health Sciences, Japan
}

\begin{abstract}
Purpose] The purpose of this study was to clarify the impact of postural changes during tidal breathing on the configuration and motion of chest-wall in order to further breathing motion evaluation. [Subjects and Methods] Chest-wall configuration and motion in the supine, right lateral, and sitting positions were measured using optoelectronic plethysmography in 15 healthy adult men. [Results] The anteroposterior diameters of the chest wall were significantly lower in the supine position for the pulmonary and abdominal rib cages, whereas the mediolateral diameters in the lateral position were lowest for the abdominal rib cage. Regarding chest-wall motion, both craniocaudal and anteroposterior motions of the anterior surface of the pulmonary and abdominal rib cages were significantly greater in the sitting position. Regarding motion of the left lateral abdominal rib cage, lateral motion was greatest in the lateral position. [Conclusion] Chest-wall configuration and motion changed according to posture in healthy men, particularly in the pulmonary and abdominal rib cages.

Key words: Chest-wall configuration, Chest-wall motion, Posture
\end{abstract}

(This article was submitted Jul. 14, 2016, and was accepted Sep. 20, 2016)

\section{INTRODUCTION}

Breathing motion of the chest wall in humans involves both the rib cage and abdomen $(\mathrm{AB})^{1)}$. Because the functional characteristics of each site differ, studies on ventilation dynamics are generally conducted according to these individual sites $^{2)}$. Furthermore, there is a pressure gradient in both the rib cage and $\mathrm{AB}^{3,4)}$ and it is believed that, along with anatomical characteristics $^{5}$, breathing-associated motion of the rib cage and $\mathrm{AB}$ varies according to each local area ${ }^{6}$. For example, in the upper rib cage, a pump-handle motion is observed, whereas in the lower rib cage, a bucket-handle motion is observed ${ }^{5,7)}$. The methods used to study these motions include a calculation model $^{2,7)}$ and radiography ${ }^{5}$; however, there have been no studies on chest-wall motion of the actual body surface.

A typical method for the detailed examination of chest-wall motion is optoelectronic plethysmography $(\mathrm{OEP})^{8)}$. This method involves dividing the chest wall into three areas, the pulmonary rib cage (RCp), abdominal rib cage (RCa), and $\mathrm{AB}$, and then calculating volume changes at each site ${ }^{9}$. However, few reports have thoroughly examined the magnitude of chest motion at each location, i.e., the anterior, dorsal, and lateral surfaces ${ }^{6}$. In particular, posture has been reported to affect chest-wall motion ${ }^{10-12)}$, and it is likely that local motion in the chest wall changes in various postures; however, De Groote et al. only reported results for the sitting posture. Some reports examined local chest-wall motion in various postures using magnetometry ${ }^{10)}$. However, unlike OEP, magnetometry is unable to identify motion direction, and the measurable sites are limited; therefore, it is difficult to ascertain chest-wall motion comprehensively using this method. In many situations, such

\footnotetext{
*Corresponding author. Sachie Takashima (E-mail: sachie@konan-wu.ac.jp)

(C)2017 The Society of Physical Therapy Science. Published by IPEC Inc.

This is an open-access article distributed under the terms of the Creative Commons Attribution Non-Commercial No Derivatives (by-nc-nd) License $<$ http://creativecommons.org/licenses/by-nc-nd/4.0/>.
} 
as the early postoperative period, clinical evaluation has to be performed in the supine position; however, this can be difficult on a daily basis using the aforementioned equipment. On the other hand, clarification of changes in chest-wall movement according to each posture may be important for the accurate assessment of changes in chest-wall motion as a result of disease.

The purpose of the present study was to examine the effects of posture on the diameter of the chest wall, and the horizontal configuration, motion direction, and magnitude of motion for each local area. To this end, we measured chest-wall motion during breathing at rest using OEP in healthy men placed in supine, lateral recumbent, and sitting positions.

\section{SUBJECTS AND METHODS}

The study included 15 healthy men without respiratory dysfunction. Table 1 shows the age and body composition values of the subjects. All subjects were provided with a full explanation prior to the study, and the study was performed after obtaining written consent. The study was performed with the approval of the ethical review board of Konan Women's University.

Chest-wall motion measurements were performed using OEP with eight cameras (Mac 3D System, Motion Analysis Corporation, San Diego, CA, USA). Reflective markers (9 $\mathrm{mm}$ in diameter) were pasted on the surface of the subject's body. In accordance with previous studies, the reflective markers were pasted on 66 sites in the supine position, 81 sites in the right lateral recumbent position (lateral recumbent position), and 86 sites in the sitting position ${ }^{8,12)}$. Furthermore, the supine and lateral recumbent positions were on the floor, and, for the lateral recumbent position, the subject was instructed to prop the left upper arm on a cushion placed on the greater trochanter in order to support the $\operatorname{arm}^{12)}$.

In all patients, measurements were recorded for 2 min during breathing at rest in each position, and the order of the measurements was randomized. For each position, the tidal volume was measured during breathing at rest using a hot-wire flow meter (AE300-s, Minato Medical Science, Tokyo, Japan) and then synchronized with the chest-wall motion for input into the analysis software. To avoid measuring motion not associated with breathing, all patients were instructed not to alter their posture during measurements. The coordinate data of each marker were entered into the analysis software (EVaRT5.04, Motion Analysis Corporation) at a sampling frequency of $100 \mathrm{~Hz}$. Thereafter, the breaths in the last $20 \mathrm{~s}$ of each measurement were extracted for analysis, and then, the chest-wall configuration and breathing motion were analyzed. In accordance with the method of Vellody et al. ${ }^{10)}$, chest-wall configuration analysis involved calculation of the anteroposterior diameters of the $\mathrm{RCp}$, anteroposterior diameters of the $\mathrm{RCa}$, and anteroposterior and mediolateral diameters of the $\mathrm{AB}$ at the end-expiratory lung volume (EELV) of breathing at rest in the positions shown in Fig. 1. Furthermore, on the basis of the coordinate values obtained by OEP, we created a horizontal cross-sectional view of the chest wall in the same position as Fig. 1 using the image-processing software gnuplot 4.4 (http://www.gnuplot.info/), and visually compared the configurations.

Chest-wall motion analysis involved calculating the coordinate variation associated with breathing in the craniocaudal, mediolateral, and anteroposterior directions for each reflective marker. Coordinate variations in each direction were included if the chest wall could be measured in all positions, and the mean value was calculated according to the following five sites: anterior RCp (14 markers; A-RCp), anterior RCa (6 markers; A-RCa), left RCa (6 markers; L-RCa), anterior AB (12 markers; $\mathrm{A}-\mathrm{AB}$ ), and left $\mathrm{AB}$ (6 markers; L-AB) (Fig. 2). Using the image-processing software gnuplot 4.4 (http://www.gnyplot. info/), we created a three-dimensional model of the chest wall in each position for all patients and represented chest-wall motion during breathing at rest as a vector. Chest-wall configurations and vectors were visually examined in the horizontal and sagittal planes.

Data are shown as means \pm standard deviations. Study data included tidal volume, changes in chest-wall diameter, and mean coordinate variation, which were examined using one-way ANOVA in order to determine how they were affected by posture, and in the event that a significant difference was observed, a post-hoc test was performed using the Bonferroni method. All statistical tests were performed using SPSS version 20.0 for Windows (SPSS Inc., Chicago, IL, USA), and a p-value of $<0.05$ was considered significant.

\section{RESULTS}

The tidal volumes in the supine, lateral recumbent, and sitting positions were $0.52 \pm 0.15,0.51 \pm 0.12$, and $0.56 \pm 0.20 \mathrm{~L}$, respectively, and there was no effect of posture on tidal volume.

Table 2 shows that the anteroposterior diameters of the RCp and RCa were significantly lower in the supine position than in the sitting and lateral recumbent positions $(\mathrm{p}<0.001)$ in EELV, whereas the mediolateral diameter of the RCa was significantly lower in the lateral recumbent position than in the supine and sitting positions $(\mathrm{p}<0.001)$. The AB anteroposterior diameters significantly increased in the following order: supine position, lateral recumbent position, and sitting position $(\mathrm{p}<0.001)$. The mediolateral diameter was significantly lower in the lateral recumbent position than in the supine and sitting positions $(\mathrm{p}<0.001)$.

Next, the horizontal configurations of the RCp, RCa, and AB were visually compared (Fig. 3). In the front of the chest wall in the supine position, the mediolateral diameters, in contrast to the anteroposterior diameters, presented larger elliptical shapes for the $\mathrm{RCp}, \mathrm{RCa}$, and $\mathrm{AB}$, whereas the dorsal side showed a mediolateral symmetrical shape in contact with the floor surface. In the lateral recumbent position, the anteroposterior diameters of the RCp, RCa, and $\mathrm{AB}$ increased, and the anteroposterior diameters and mediolateral diameters presented approximately equal shapes. Furthermore, a decrease in 
Table 1. Subject demographics and anthropometric parameters

\begin{tabular}{lr}
\hline Age (years) & $27.5 \pm 4.6$ \\
Weight $(\mathrm{kg})$ & $66.9 \pm 8.9$ \\
Height $(\mathrm{cm})$ & $175 \pm 7.1$ \\
BMI $\left(\mathrm{kg} / \mathrm{m}^{2}\right)$ & $21.8 \pm 2.4$ \\
\hline
\end{tabular}

BMI: body mass index, All data are expressed as means $\pm \mathrm{SD}$

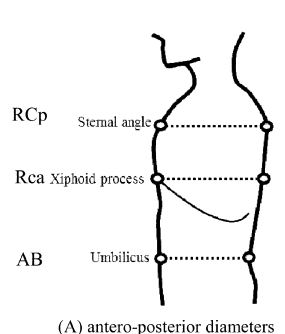

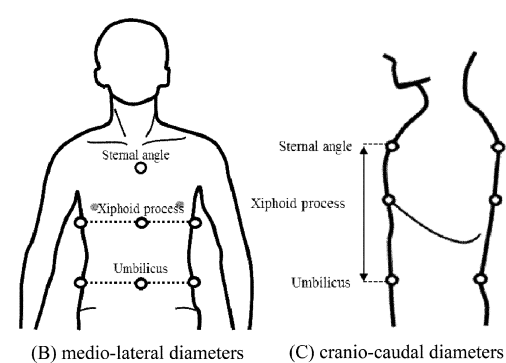

$\begin{array}{lll}\text { (B) medio-lateral diameters } & \text { (C) cranio-caudal diameters }\end{array}$
Fig. 1. Representation of the chest wall using external markers: model used to calculate the anteroposterior (A), mediolateral (B), and craniocaudal diameters (C)

$\mathrm{RCp}$ : pulmonary rib cage, $\mathrm{RCa}$ : abdominal ribcage, $\mathrm{AB}$ : abdomen

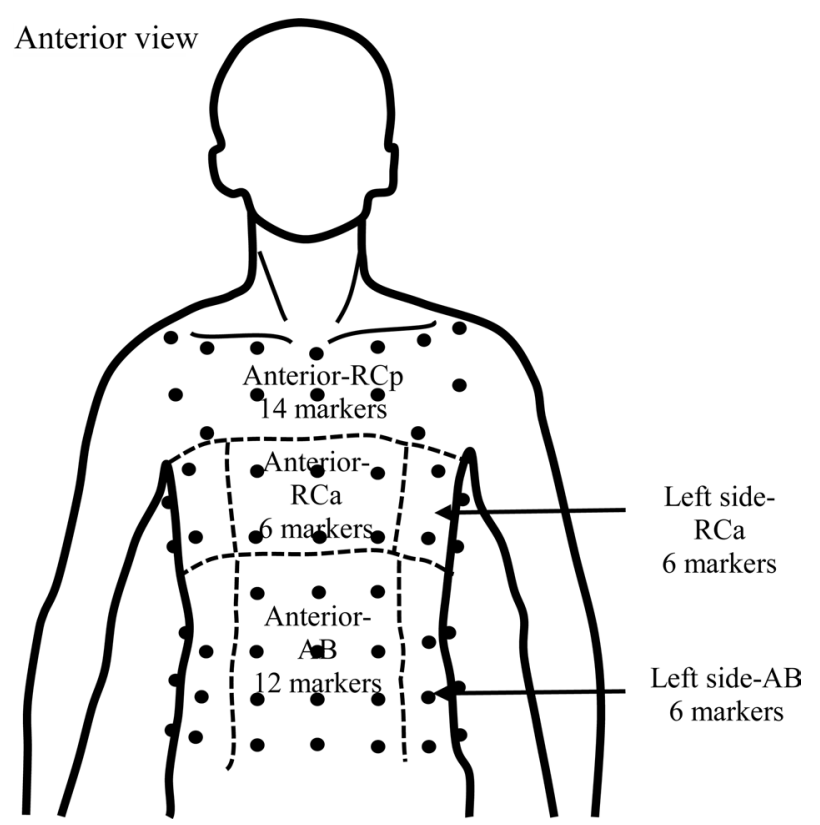

Fig. 2. Division of the chest wall into compartments and placement of passive reflective markers on the chest wall: RCp: pulmonary rib cage, $\mathrm{RCa}$ : abdominal rib cage, $\mathrm{AB}$ : abdomen

The chest wall was divided into 5 parts: Anterior-RCp (14-markers), Anterior-RCa (6-markers), Left side-RCa (6-markers), Anterior- $\mathrm{AB}$ (12-markers), and Left side- $\mathrm{AB}$ (6-markers)

Table 2. Chest-wall diameter changes during tidal breathing in three postures (EELV) [mm]

\begin{tabular}{lllll}
\hline & & Supine & Lateral & Sitting \\
\hline RCp & antero-posterior & $168.8 \pm 11.2$ & $206.0 \pm 15.3 *$ & $201.0 \pm 13.0^{*}$ \\
RCa & antero-posterior & $192.6 \pm 21.9$ & $237.8 \pm 23.1 *$ & $223.7 \pm 22.9^{*}$ \\
& medio-lateral & $325.1 \pm 13.5+$ & $282.8 \pm 14.3 *$ & $323.5 \pm 14.6+$ \\
AB & antero-posterior & $181.1 \pm 20.4$ & $225.4 \pm 28.9 *$ & $271.5 \pm 19.4 *+$ \\
& medio-lateral & $310.6 \pm 22.4$ & $271.5 \pm 19.4 *$ & $312.0 \pm 22.6+$ \\
\hline
\end{tabular}

Expressed as means $\pm \mathrm{SD}$. ${ }^{*} \mathrm{p}<0.01$ vs. supine $+\mathrm{p}<0.01$ vs. lateral

the mediolateral diameter of the right side, i.e., the weight-bearing side, was observed in the $\mathrm{RCa}$ and $\mathrm{AB}$, presenting an asymmetrical shape with respect to the left side. Moreover, we found that the midline of the sternal angle and xiphoid process was rotated to the left relative to the umbilicus. The chest wall does not come into contact with the surface of the floor in the sitting position, and therefore, a rounded elliptical shape was presented on both the anterior and posterior sides, and, as with the supine position, the mediolateral diameters showed a large symmetrical shape.

Regarding motion of the anterior RCp, craniocaudal motion was significantly greater in the sitting position than that in the lateral recumbent and supine positions ( $\mathrm{p}<0.01$ and $\mathrm{p}<0.05$, respectively) (Table 3 , Fig. 4). Medio-lateral motion showed a significant change in the sitting position compared with that in the lateral recumbent position $(\mathrm{p}<0.01)$, and anteroposterior motion was significantly greater in the sitting position than that in the supine and lateral recumbent positions $(p<0.01)$.

Next, in the sagittal plane of the sitting position (Fig. 4), the RCp vector was shown to be large in the upper anterior area, and, therefore, compared with that in the supine and lateral recumbent positions, in the RCp of the sitting position, chest-wall motion occurred in the upper anterior area.

Regarding motion on the anterior side of the $\mathrm{RCa}$, craniocaudal motion was significantly greater in the sitting position than in the lateral recumbent and supine positions ( $p<0.01$ and $p<0.05$, respectively). Similarly, anteroposterior motion was significantly greater in the sitting position than in the lateral recumbent and supine positions $(\mathrm{p}<0.01)$. However, mediolateral motion showed a significant change in the lateral recumbent position compared to the sitting position $(\mathrm{p}<0.05)$.

Regarding RCa motion on the left side, i.e., the non-weight-bearing side, craniocaudal motion was significantly greater in the sitting position than in the supine and the lateral recumbent positions $(\mathrm{p}<0.01)$, whereas mediolateral motion was signifi- 


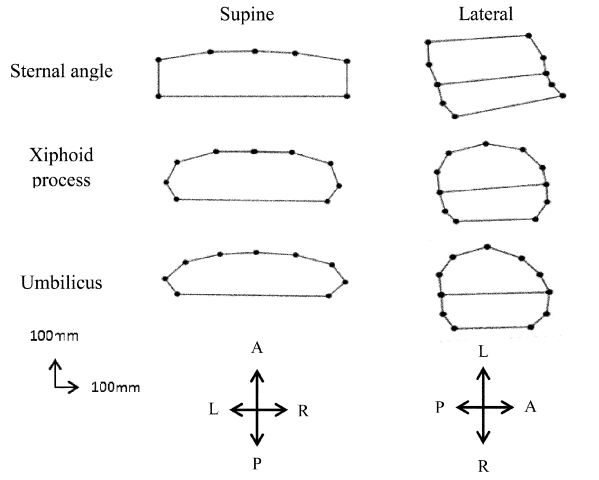

Fig. 3. Computed models of chest-wall configuration (horizontal plane) for all subjects (A: anterior, P: posterior, R: right, L: left)

Left fig.: supine position, Center fig.: right lateral position, Right fig.: sitting position

The black dots represent markers and the center line of the chest wall divides the chest into right and left.

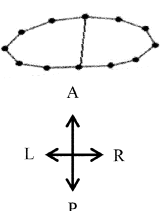

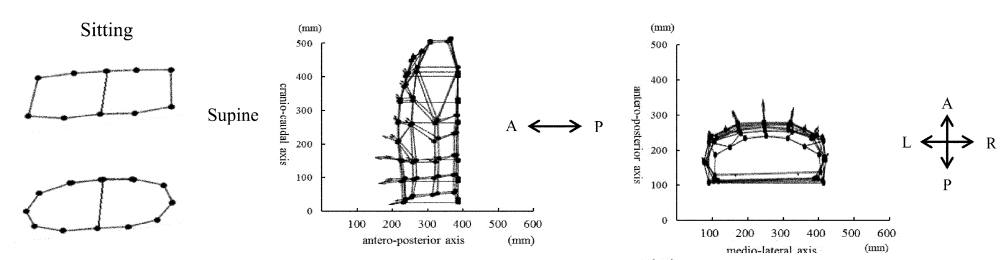
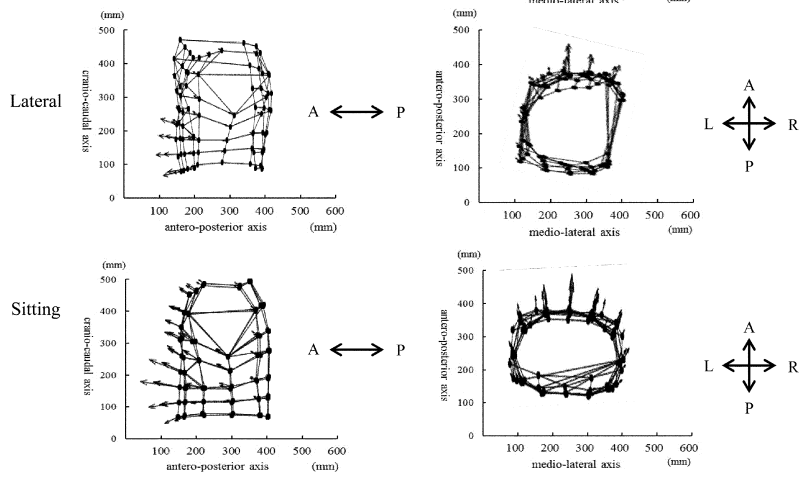

Fig. 4. Computed models of the chest wall and mean tidal vectors for all subjects (A: anterior, P: posterior, R: right, L: left) Left fig.: sagittal plane of chest wall (left side) Right fig.: horizontal plane of chest wall (cranial side)

The tidal vectors were transcribed in triple size.

Table 3. Mean chest-wall coordinates during tidal breathing in three postures [mm]

\begin{tabular}{clccc}
\hline & & Supine & Lateral & \multicolumn{1}{c}{ Sitting } \\
\hline \multirow{3}{*}{ A-RCp } & cranio-caudal & $1.14 \pm 0.68$ & $0.68 \pm 0.34$ & $1.94 \pm 1.07^{*++}$ \\
& medio-lateral & $-0.03 \pm 0.19$ & $0.29 \pm 0.29$ & $-0.34 \pm 0.53++$ \\
& antero-posterior & $0.69 \pm 0.73$ & $0.64 \pm 0.81$ & $1.91 \pm 1.37^{* *++}$ \\
& cranio-caudal & $1.69 \pm 0.61$ & $1.03 \pm 0.37$ & $2.58 \pm 1.26^{*++}$ \\
A-RCa & medio-lateral & $0.11 \pm 0.23$ & $-0.29 \pm 0.39$ & $0.26 \pm 0.78+$ \\
& antero-posterior & $1.50 \pm 0.73$ & $0.94 \pm 0.53$ & $2.81 \pm 1.14^{* *++}$ \\
& cranio-caudal & $1.08 \pm 0.45$ & $0.95 \pm 0.40$ & $2.19 \pm 0.94^{* *++}$ \\
L-RCa & medio-lateral & $0.41 \pm 0.44$ & $1.70 \pm 0.58^{* *}$ & $0.96 \pm 0.85+$ \\
& antero-posterior & $1.48 \pm 0.52$ & $1.65 \pm 0.88$ & $2.11 \pm 1.09$ \\
& cranio-caudal & $0.73 \pm 0.60$ & $0.54 \pm 0.73$ & $0.93 \pm 0.93$ \\
A-AB & medio-lateral & $0.15 \pm 0.23$ & $-0.54 \pm 0.68^{*}$ & $0.32 \pm 1.04++$ \\
& antero-posterior & $5.55 \pm 1.83$ & $5.23 \pm 1.66$ & $4.79 \pm 2.60$ \\
& cranio-caudal & $0.23 \pm 0.20$ & $-0.11 \pm 0.33^{*}$ & $0.58 \pm 0.37^{*++}$ \\
L-AB & medio-lateral & $0.77 \pm 0.40$ & $1.17 \pm 0.50$ & $0.55 \pm 0.61++$ \\
& antero-posterior & $1.45 \pm 0.42$ & $1.25 \pm 0.63$ & $1.59 \pm 0.73$ \\
\hline A: Anter
\end{tabular}

A: Anterior, L: Left, craniocaudal: coordinate of vertical axis, mediolateral: coordinate of the forehead axis, anteroposterior: coordinate of the sagittal axis. The coordinate change to the upper left in the locus in front was defined as plus. Expressed as means \pm SD $* \mathrm{p}<0.05, * * \mathrm{p}<0.01$ vs. supine $+\mathrm{p}<0.05,++\mathrm{p}<0.01$ vs. lateral

cantly greater in the lateral recumbent position than in the supine and the sitting positions $(\mathrm{p}<0.01$ and $\mathrm{p}<0.05$, respectively); however, no significant differences were observed between postures with respect to anteroposterior motion.

As with the RCp, in the sitting sagittal plane (Fig. 4), the RCa vector was shown in the upper anterior area. However, for the left side in the horizontal plane in the lateral recumbent position, we observed motion toward the left lateral side, and we confirmed a trend different from that of chest-wall motion direction at the same site in the sitting position.

Regarding motion on the anterior of the $\mathrm{AB}$, in the craniocaudal and anteroposterior directions, no significant differences were observed between the postures, whereas for mediolateral motion, significant changes were observed in the lateral recumbent position compared to the supine $(\mathrm{p}<0.05)$ and sitting positions $(\mathrm{p}<0.01)$. 
Regarding motion on the left lateral side, i.e., the non-weight-bearing side of the $\mathrm{AB}$, there was significantly less craniocaudal motion in the lateral recumbent position compared to the supine position $(p<0.05)$. Furthermore, craniocaudal motion in the sitting position was significantly greater in the sitting position than that in the supine and lateral recumbent positions $(\mathrm{p}<0.05$ and $\mathrm{p}<0.01$, respectively). Mediolateral motion was significantly greater in the lateral recumbent position than in the sitting position $(\mathrm{p}<0.01)$. As with $\mathrm{RCa}$, there was no difference in anteroposterior motion between postures.

In the sagittal plane shown in Fig. 4, anterior chest-wall motion was confirmed in all postures, and there was no difference observed between postures. However, in the horizontal plane, a mediolateral symmetrical vector was shown in the supine and sitting positions, whereas in the lateral recumbent position, the vector for the right side, i.e., the weight-bearing side, was more asymmetrical than the vector for the left side.

\section{DISCUSSION}

In the present study, we examined the effects of posture on the configuration and motion of the chest wall in healthy men. Regarding configuration, the anteroposterior diameters of the RCp and RCa were smallest in the supine position, the mediolateral diameter of the RCa was smallest in the lateral recumbent position, and the chest wall tended to decrease in the direction of the force of gravity. Furthermore, our analysis of chest-wall motion during breathing at rest revealed that craniocaudal and anteroposterior motions of the RCp and RCa were characteristically greatest in the sitting position and that mediolateral motion was greatest in the lateral recumbent position.

To date, several reports have examined the effect of posture on chest-wall motion. Vellody et al. ${ }^{10)}$ examined the configuration and motion of the chest wall in the supine, lateral recumbent, and sitting positions using magnetometry. They reported that in the direction of gravity, chest-wall configuration reduced in size and that breathing motion was predominantly abdominal in the supine position compared to the sitting position. Furthermore, Verschakelen et al. ${ }^{11)}$ examined changes in the chest-wall diameter in the supine, sitting, and standing positions using respiratory inductance plethysmography (RIP) and reported that breathing motion at rest tended to be predominantly abdominal in the supine position compared to the standing position. However, both the magnetometry and RIP devices in these studies are characteristically incapable of examining chest-wall motion. Similar to our study, De Groote A used OEP to examine the direction and magnitude of breathing motion at localized sites of the chest wall. Their results revealed that for the rib cage, breathing motion was greater in the craniocaudal and anteroposterior directions, whereas for the $\mathrm{AB}$, breathing motion was mainly in an anteroposterior direction. However, the number of reflective markers used in their study was $\leq 36$ and the measurement position was limited to the sitting position ${ }^{6}$.

In our study, regarding EELV, the anteroposterior diameters of the $\mathrm{RCp}, \mathrm{RCa}$, and $\mathrm{AB}$ were smallest in the supine position. The mediolateral diameters of the $\mathrm{RCa}$ and $\mathrm{AB}$ were small in the lateral recumbent position, and the anteroposterior diameter of the $\mathrm{AB}$ was largest in the sitting position. Although the measurement method differed, our results were comparable to those reported by Vellody et al. ${ }^{10)}$, probably because, despite using OEP, when calculating coordinate values of the chest-wall diameter, we calculated the distance between two typical markers.

However, in the present study, we were able to obtain the characteristics of chest-wall configuration as shown in Fig. 3 using a novel method. We found that the anteroposterior diameters were smallest in the supine position with a mediolateral symmetrical and elliptical shape, whereas in the lateral recumbent position, the anteroposterior diameters were large with small mediolateral diameters and round shapes, indicating that the mediolateral chest-wall configuration was asymmetrical. Regarding the changes in configuration observed here, it is believed that changing to the lateral recumbent position may have caused the mediastinum to shift in a sagittal plane direction ${ }^{6,12)}$. On the basis of the results of the AB diameter and horizontal plane configuration, it is believed that changes in the direction of the force of gravity along with changes in posture cause a shift in the abdominal organs, which may expand the abdominal wall and change the configuration ${ }^{14)}$

The most significant finding of the present study is that local chest-wall motion associated with breathing is affected by posture. To date, it has been said that the local breathing motion of the chest wall in the sitting position tends to be in a craniocaudal and anteroposterior direction for the rib cage ${ }^{6}$.

However, the present study revealed that in postures, including the supine and lateral recumbent positions, such breathing motion was reduced. Furthermore, similar to De Groote et al. ${ }^{6}$, regarding the sitting position, we found that mediolateral motion of the rib cage was much less, whereas in the lateral recumbent position, mediolateral motion was more, and, in particular, mediolateral motion of the non-weight-bearing side of the abdominal rib cage was greatest in the lateral recumbent position $(1.704 \pm 0.578 \mathrm{~mm})$. Previously, in the lateral recumbent position, the change in chest-wall volume associated with breathing was found to be characteristically greater for the abdominal rib cage of the non-weight-bearing side ${ }^{12)}$, supporting our results in the present study. Although the effect of posture on these rib cage breathing motions cannot be clarified by the results of the present study alone, we believe that the mediolateral asymmetry of the chest wall in the lateral recumbent position may have been caused by local pressure on the chest wall due to the floor surface ${ }^{12)}$, in addition to factors indicated by Vellody et al. ${ }^{10)}$, which include 1) difference in respiratory muscle action distribution, 2) change in the mechanical advantage of the inspiratory muscles, and 3) change in local compliance. Furthermore, on the basis of our result indicating that the midline rotated to the left at the level of the sternal angle and xiphoid process in the lateral recumbent position (Fig. 4), it is believed that the angle made by the ribs and vertebral body affects rib motion ${ }^{5,6)}$ and that changes in the costovertebral joint angle associated with changes in posture possibly caused the mediolateral diameters to change. 
Breathing motion of the $\mathrm{AB}$ has been found to be characteristically greater in the supine position than in the sitting and standing positions ${ }^{11)}$. Furthermore, De Groote ${ }^{6}$ reported that abdominal motion in the sitting position was mainly in an anteroposterior direction. However, our results demonstrated that abdominal motion was not affected by posture, and, in all postures, the motion was greater in the anteroposterior direction. As mentioned by Konno et al. ${ }^{15)}$, this could be attributed to the effect of differences in compliance between the $\mathrm{AB}$, which consists of soft tissue, and the chest, which is supported by osseous structures such as the sternum and ribs. Furthermore, on comparing local motion of the AB, in the present study, we observed no site at which motion was greatest in the supine position. Therefore, differences in our study compared to results of earlier studies could have been caused by differences in the measurement methods. Although only part of the chest wall can be measured using conventional methods (magnetometry and RIP), with our method, we were able to measure the entire chest wall, and we believe that this explains why we found no such differences in the present study.

Chest-wall motion tends to vary in patients after surgery, and in patients with severe chronic obstructive pulmonary disease $^{13,16)}$, it is quite difficult to measure chest-wall volume in clinical practice. In such instances, local chest-wall motion must be assessed by visual examination and palpation. We believe that our results could be used as a healthy reference, and, in particular, understanding which sites move the most according to posture (craniocaudal and anteroposterior direction of the rib cage in the sitting position, and mediolateral motion of the non-weight-bearing side of the abdominal rib cage in the lateral recumbent position) will help detect abnormalities in breathing motion at each site.

A limitation of the present study was that only men were included. Chest-wall motion differs between men and wom$\mathrm{en}^{11,14,17)}$, and, therefore, the results of the present study cannot be applied to women. Furthermore, in the present study, measurements were recorded with subjects on the floor in order to avoid the possibility of the chest wall sinking into the mattress if a bed had been used. Therefore, our results cannot necessarily be applied to patients who are bedridden.

In the present study, we examined the effect of posture on chest-wall configuration and breathing motion at various sites in healthy men.

The results revealed that the anteroposterior diameters of the RCp and RCa were smallest in the supine position, the mediolateral diameter of the RCa was smallest in the lateral recumbent position, and the chest wall tended to decrease in the direction of gravitational force.

Regarding breathing motion, in the supine and lateral recumbent positions, the motion of the rib cage decreased in the craniocaudal and anteroposterior directions, and in the lateral recumbent position, mediolateral motion in particular was greater. Furthermore, abdominal breathing motion was hardly influenced by posture. Therefore, the chest-wall configuration and local motion were affected by posture and the effects were particularly notable in the rib cage.

\section{REFERENCES}

1) Ward ME, Ward JW, Macklem PT: Analysis of human chest wall motion using a two-compartment rib cage model. J Appl Physiol 1985, 1992 , 72: $1338-1347$. [Medline]

2) Chihara K, Kenyon CM, Macklem PT: Human rib cage distortability. J Appl Physiol 1985, 1996, 81: 437-447. [Medline]

3) Tawhai MH, Nash MP, Lin CL, et al.: Supine and prone differences in regional lung density and pleural pressure gradients in the human lung with constant shape. J Appl Physiol 1985, 2009, 107: 912-920. [Medline] [CrossRef]

4) De Keulenaer BL, De Waele JJ, Powell B, et al.: What is normal intra-abdominal pressure and how is it affected by positioning, body mass and positive endexpiratory pressure? Intensive Care Med, 2009, 35: 969-976. [Medline] [CrossRef]

5) Wilson TA, Rehder K, Krayer S, et al.: Geometry and respiratory displacement of human ribs. J Appl Physiol 1985, 1987, 62: 1872-1877. [Medline]

6) De Groote A, Wantier M, Cheron G, et al.: Chest wall motion during tidal breathing. J Appl Physiol 1985, 1997, 83: 1531-1537. [Medline]

7) Saumarez RC: An analysis of possible movements of human upper rib cage. J Appl Physiol 1985, 1986, 60: 678-689. [Medline]

8) Cala SJ, Kenyon CM, Ferrigno G, et al.: Chest wall and lung volume estimation by optical reflectance motion analysis. J Appl Physiol 1985, 1996, 81: 26802689. [Medline]

9) Kenyon CM, Cala SJ, Yan S, et al.: Rib cage mechanics during quiet breathing and exercise in humans. J Appl Physiol 1985, 1997, 83: 1242-1255. [Medline]

10) Vellody VP, Nassery M, Druz WS, et al.: Effects of body position change on thoracoabdominal motion. J Appl Physiol, 1978, 45: 581-589. [Medline]

11) Verschakelen JA, Demedts MG: Normal thoracoabdominal motions. Influence of sex, age, posture, and breath size. Am J Respir Crit Care Med, 1995, 151: 399-405. [Medline] [CrossRef]

12) Nozoe M, Mase K, Takashima S, et al.: Measurements of chest wall volume variation during tidal breathing in the supine and lateral positions in healthy subjects. Respir Physiol Neurobiol, 2014, 193: 38-42. [Medline] [CrossRef]

13) De Groote A, Van Muylem A, Scillia P, et al.: Ventilation asymmetry after transplantation for emphysema: role of chest wall and mediastinum. Am J Respir Crit Care Med, 2004, 170: 1233-1238. [Medline] [CrossRef]

14) Romei M, Mauro AL, D'Angelo MG, et al.: Effects of gender and posture on thoraco-abdominal kinematics during quiet breathing in healthy adults. Respir Physiol Neurobiol, 2010, 172: 184-191. [Medline] [CrossRef]

15) Konno K, Mead J: Measurement of the separate volume changes of rib cage and abdomen during breathing. J Appl Physiol, 1967, 22: 407-422. [Medline]

16) Binazzi B, Bianchi R, Romagnoli I, et al.: Chest wall kinematics and Hoover's sign. Respir Physiol Neurobiol, 2008, 160: 325-333. [Medline] [CrossRef]

17) Vogiatzis I, Aliverti A, Golemati S, et al.: Respiratory kinematics by optoelectronic plethysmography during exercise in men and women. Eur J Appl Physiol, 2005, 93: 581-587. [Medline] [CrossRef] 